\title{
La evaluación formativa y compartida en la Formación Inicial del Profesorado desde la perspectiva del alumnado y de los egresados
}

\author{
Carolina Hamodi Galán ${ }^{1 *}$ y Ana Teresa López Pastor ${ }^{2}$ \\ ${ }^{1}$ Escuela Universitaria de Educación de Soria \\ ${ }^{2}$ Facultad de Ciencias Sociales, Jurídicas y de la Comunicación de Segovia, Universidad de \\ Valladolid
}

(Received September 20, 2011; Accepted January 25, 2012)

RESUMEN: Este artículo presenta la valoración de alumnos y egresados de Educación Superior (Formación Inicial del Profesorado), sobre la evaluación formativa y compartida. La investigación se realizó con dos grupos de discusión, uno con alumnos de último curso y otro con egresados de los últimos tres años, por ser generaciones que habían teniendo en alguna o varias asignaturas este modelo evaluativo. En ambos grupos se contó con alumnos y alumnas de todas las especialidades impartidas en la Escuela de Magisterio. Los grupos de discusión se realizaron en la primavera de 2009 y el análisis temático de los discursos se hizo con el software cualitativo Atlas.ti 6.0.

Son cinco las conclusiones extraídas de sus discursos sobre las que reflexionamos: (1) consenso claro en que la evaluación formativa ayuda a aprender más y mejor; (2) consideración de la evaluación como algo útil para educar; (3) convicción de que participar da valor al modelo, aceptándose más la evaluación colaborativa con el profesor que entre iguales; (4) demanda de coherencia entre el discurso pedagógico y la práctica educativa; y (5) petición de formación continua para aplicar lo experimentado a su práctica docente, empezando por el prácticum.

A partir de los resultados consideramos necesario seguir profundizando en los discursos de los alumnos, especialmente en el de los menos favorables al modelo, con el fin de mejorarlo. Resultaría también interesante recorrer dos nuevos caminos que indican los discursos analizados: abrir líneas de investigación-acción respecto al prácticum, y contrastar las posibilidades de establecer redes para una formación continua.

Palabras clave: evaluación formativa, evaluación compartida, docencia universitaria, formación inicial del profesorado, alumnado, egresados.

\section{Formative and shared assessment in the Pre-service teacher education} from the perspective of students and graduates

\footnotetext{
ABSTRACT: This paper presents the assessment of students and graduates of higher education (initial teacher training) on formative assessment and shared. The research was conducted with two discussion groups with students a thirdyear graduates and another for the last three years because it had given genera- 
tions in one or more of their subjects this evaluation model. Both groups had men and women of all specialties taught at the School of Education. The discussion groups were conducted in the spring of 2009 and the theme of discourse analysis was done qualitative software Atlas.ti 6.0.

Five are the conclusions drawn from his speeches on which we reflect: (1) Clear consensus that formative assessment helps you learn more and better; (2) consideration of the evaluation as something useful to educate; (3) the conviction that participation gives value to the model, accepting more collaborative assessment with the teacher to peer; (4) demand for consistency between discourse and educational practice teaching; and (5) request for trainning to implement anything experienced in their teaching practice, beginning with the practicum. We believe, based on the results, which must continue to deepen students' speeches, especially in the less favorable of the model, in order to improve it. It would also be interesting to explore two new roads that indicate the analyzed speeches: to open new lines of research-action on the practicum, and contrast the networking possibilities for training.

Keywords: formative assessment, shared assessment, higher education, preservice teacher education, students, graduates.

\section{INTRODUCCIÓN. CONSIDERANDO LA VOZ DEL ALUMNADO Y DE LOS EGRESADOS EN LA EVALUACIÓN FORMATIVA Y COMPARTIDA}

Los debates en torno a la evaluación formativa y compartida en la Formación Inicial del Profesorado vienen reflexionando sobre sus ventajas e inconvenientes. Este artículo aporta como novedad su valoración desde los discursos de los propios alumnos y egresados (en este caso de Magisterio de Segovia), a través de una técnica cualitativa: los grupos de discusión.

Utilizar un sistema de evaluación formativa y compartida, en lugar de un modelo más tradicional, tiene una serie de repercusiones para el alumnado. Desde la bibliografía se señalan sus numerosos efectos positivos así como sus inconvenientes dentro de su aplicación en el contexto del EEES, pero en la mayoría de los casos se describen por los profesores y desde su punto de vista mediante diferentes técnicas: revisiones bibliográficas (Bretones, 2008; Dochy, Segers y Dierick, 2002), estudio de casos a partir de autoinformes de profesores (Buscá, Pintor, Martínez y Peire, 2010; Gómez y Grau, 2010; López, 2009), ciclos de investigación-acción (Capllonch, Buscà, Martín, Martínez y Camerino, 2008; Pérez et al., 2008; Vallés, Ureña y Ruiz, 2011) o entrevistas (Gros y Romaña, 1995; Tejedor, 1998).

Consideramos que para hablar desde la perspectiva del alumnado ha de tenerse en cuenta su percepción. Algunos estudios se centran en los cuestionarios a los discentes como técnica para obtener esta información (Álvarez, García, Gil, Romero y Correa, 2002; Arribas, Carabias y Monreal, 2010; Ibarra y Rodríguez, 2007; Palomares, 2011; Porto, 2009; Tejedor, 1998), o en el análisis de las evidencias del alumnado (Palomares, 2011; Ros, 2001), pero consideramos 
que a pesar de que tiene una serie de ventajas (puede aplicarse a una muestra más grande), se pierden muchos matices interesantes que pueden obtenerse de su discurso, que pueden ser muy relevantes para mejorar el sistema de evaluación formativa y compartida. Hasta el momento son escasos los informes a partir de grupos de discusión (Álvarez et al., 2002). Dentro de estas investigaciones, son muy pocos los casos en los que las citas del alumnado ven la luz. En Brown y Glasner (2003), aparecen 16 experiencias sobre evaluación formativa; solamente la experiencia recogida por McDowel y Sambell aporta citas de los argumentos expuestos por los discentes.

Reflexionar sobre los discursos de los alumnos nos puede permitir avanzar en la mejora del modelo, de cara a alcanzar un verdadero cambio de paradigma en la evaluación en la educación superior. Conscientes de ello, el objeto de estudio de esta investigación buscaba conocer la imagen (ventajas, inconvenientes) que tenían sobre la evaluación formativa y compartida los alumnos y los egresados de los últimos tres años (cuando se viene utilizando este sistema), de la Escuela de Magisterio de Segovia y las propuestas de mejora que plantearan.

Decidimos añadir al colectivo de egresados en el estudio al considerar su discurso muy interesante, pues el tiempo les aporta una perspectiva diferente, e incluso el estar fuera de la institución les da un mayor grado de libertad para expresar sus opiniones (Santos, 2000).

\section{APROXIMACIÓN METODOLÓGICA}

\section{Participantes en los grupos de discusión}

Tanto en el grupo de alumnos como de egresados era importante que fueran de ambos sexos y que estuvieran representadas las cuatro especialidades que existían entonces: Infantil, Primaria, Música y Educación Física. Varios profesores utilizaban, desde el curso 2003-2004, la evaluación formativa y compartida, siendo la especialidad de Educación Física (con asignaturas optativas que elegían alumnos de otras especialidades) la que tenía la mayoría de sus profesores especialistas en este modelo evaluativo, algo que aparece varias veces en los discursos. En general, en la escuela de Magisterio de Segovia se mantiene el contacto con los egresados, por lo que fue posible localizar a varios que tuvieran experiencia como profesores tras su graduación. Para el grupo de los alumnos se optó por los de último curso al disponer de mayor experiencia desde la que hablar.

El tamaño de los grupos osciló entre los 5 y los 7 participantes, y las variables que se consideraron relevantes dentro de cada uno de ellos son las señaladas en la tabla 1: 


\section{Tabla 1. Variables Consideradas}

\begin{tabular}{|ll|}
\hline \multirow{2}{*}{ COLECTIVO } & \multicolumn{1}{c|}{ VARIABLES } \\
Egresados & - Sexo \\
& - Especialidad cursada en la titulación de Magisterio \\
& (Infantil, Primaria, Musical, Educación Física) \\
& - Egresados de los últimos tres años \\
& - Experiencia laboral \\
& \\
Alumnos & - Sexo \\
& - Especialidad cursada en la titulación de Magisterio \\
& - Último curso \\
\hline
\end{tabular}

Fuente: elaboración propia.

\section{Técnicas de recogida de datos}

El grupo de discusión fue la técnica de investigación utilizada. Se llevaron a cabo en la primavera de 2009 y fueron grabados para su posterior transcripción. Duraron ambos en torno a hora y media y el discursó fluyó ágilmente.

\section{Técnicas de análisis de datos}

Con las transcripciones hemos llevado a cabo un análisis por categorías (Conde, 2010; Paillé y Muchielli, 2003), modelo de análisis de contenido desarrollado en los últimos años por la teoría fundamentada, base metodológica del programa de software cualitativo Atlas.ti 6.0., que es el que hemos utilizado técnicamente.

Tras una primera lectura en profundidad de los discursos, establecimos unos códigos o categorías (codes) para llevar a cabo una síntesis conceptual de las citas. Posteriormente señalamos aquellas citas (quotations) que contenían un potencial significativo para el análisis, otorgándoles su identificación alfanumérica. Para contextualizar las citas, hemos incluido tras ellas (entre paréntesis) "GE" (discursos de egresados) y "GA" (discursos de alumnos). Los códigos utilizados son coherentes con los objetivos planteados (tabla 2). 
Tabla 2. Objetivos y Categorías de Análisis en Atlas.ti

\begin{tabular}{|lll|}
\hline OBJETIVO & CATEGORÍA & SUBCATEGORÍAS \\
$\begin{array}{l}\text { Conocer las ventajas } \\
\text { que aporta la EFyC }\end{array}$ & "Ventajas" & $\begin{array}{l}\text { - Experimentación sistema de } \\
\text { evaluación alternativo } \\
\text { - Mayor aprendizaje } \\
\text { - Mejor aprendizaje }\end{array}$ \\
& & - Modelo más participativo \\
$\begin{array}{l}\text { Conocer los } \\
\text { inconvenientes y } \\
\text { dificultades }\end{array}$ & $\begin{array}{l}\text { "Incovenientes y } \\
\text { dificultades" }\end{array}$ & $\begin{array}{l}\text { - Carga de trabajo } \\
\text { - Evaluación entre iguales }\end{array}$ \\
$\begin{array}{l}\text { Obtener propuestas para } \\
\text { mejorarla }\end{array}$ & "Propuestas para & - Dicen pero no hacen \\
& mejorar" & - Sistema alternativo en el prácticum \\
\end{tabular}

Fuente: elaboración propia.

${ }^{1}$ Las siglas "EFyC" significan Evaluación Formativa y Compartida.

\section{RESULTADOS Y DISCUSIÓN}

Las principales ideas extraídas de los discursos se exponen en cinco apartados. A lo largo de todos ellos se perciben sus dos categorías de identidad social, la de alumnos y el de actuales o futuros profesores, que aparece constantemente en el devenir del habla. Las referencias al prácticum (todos lo habían realizado ya) son constantes; es su primera experiencia docente oficial y ellos la viven como un importante rito de paso.

\section{La evaluación formativa ayuda a aprender más y mejor}

En ambos grupos se valora más este modelo: “al final te llevas tus cálculos y dices: he aprendido muchas cosas" (GE), y cuestionan abiertamente las carencias de otros modelos evaluativos tradicionales, como alumnos y como profesores: "por eso digo, pero es que el niño según los criterios de evaluación sí aprobaba. Pero no se enteraba de nada, tú le preguntabas...y él no se enteraba de nada" (GA).

Dan mucha importancia a la capacidad de reflexión y de análisis crítico que se desarrolla y a la habilidad conseguida para afrontar el propio aprendizaje: "[Aprendes] a verlo todo más críticamente y a buscarte las castañas" (GE); "Esa capacidad crítica que hace reflexionar a través por ejemplo de una asignatura" (GE). Estos discursos coinciden con lo apuntado por Bretones (2008), Dochy, Segers y Sluijsmans (1999), López (2000) y López, Martínez y Julián (2007), donde se destaca que la evaluación formativa y compartida facilita la adquisición 
de competencias y el aprendizaje autónomo a lo largo de la vida.

Se considera positivo desarrollar un sistema de trabajo y evaluación más continuo, donde el esfuerzo cotidiano se ve recompensado en mayor medida que con los sistemas de evaluación tradicional, valorándose por todos la posibilidad que ofrece de autorregular los errores a lo largo del proceso de aprendizaje (Sanmartí, 2007):

Pienso que también aprendes el doble, porque yo en las asignaturas que he tenido un examen y punto, pues es que me lo he aprendido y mucho ya se me ha olvidado (afirman). En cambio, con las asignaturas que he tenido esta metodología es que ya es algo que se te queda dentro y lo vas a aplicar, vamos yo en cuanto pueda (GE).

Creemos que hay que considerar seriamente, como ellos hacen, la importancia del efecto acumulativo en el caso de que este modelo fuera generalizado. Lo explicitan poniendo como ejemplo una especialidad (Educación Física). Tanto los que la cursaron como los que eligieron alguna asignatura de ella como optativa reconocen sus aportaciones: "Yo creo que hay mucha diferencia de unos departamentos a otros" (GE); “Todo el tipo de evaluación... lo hemos visto en Educación Física" (GA).

\section{Participar da valor al modelo. La evaluación: útil para educar.}

Bretones (2008) lleva a cabo una revisión bibliográfica con el propósito de analizar la participación de los estudiantes de Educación Superior en su evaluación y afirma que dicha participación tiene ventajas de tipo intelectual (supone más capacidad crítica y de autonomía), de tipo afectivo (más motivación y auto responsabilidad) y de tipo profesional. De manera más general, López et al. (2007) afirman que mediante este sistema el alumnado valora positivamente su participación en los procesos de evaluación, otorgando un gran valor al modelo, idea que se corrobora en los discursos al manifestar que sienten al profesorado más cercano, entre otras cosas por dejarles participar en la toma de decisiones: "Llevando el sistema del que hemos estado hablando, lo primero que va a ser va a ser cercano porque das participación" (GA); "A mí me gusta mucho que te den la oportunidad, ya que se supone que durante la carrera eres una persona adulta, de elegir qué forma de evaluación quieres para ti ante esa asignatura, y eso me gustó mucho" (GE).

Relacionado con ello se reconoce en los discursos la importancia del modelo evaluativo como modelo a su vez educativo: “Además, esto de la evaluación es útil para educar" (GA); "Yo estoy con ella, creo que el trabajo y el esfuerzo es lo que educa, está clarísimo" (GA).

La consideración del esfuerzo realizado durante el proceso de aprendizaje es clave, viéndola así como una evaluación más justa, en parte por disponer de más oportunidades para mejorar. Reconocen, como consecuencia lógica, haber obte- 
nido mejores "notas". Dochy et al. (1999) ya concluían que los estudiantes que practican la autoevaluación tienden a puntuar más alto en los exámenes.

Aprendes más y además, por lo general, hasta sueles sacar, si se mira la nota de la carrera, se suele sacar hasta mejor nota. Porque tú mismo y a lo mejor la gente se implica más y termina sacando más nota y tal. Y jo pues ¿qué más puedes pedir? Si en esta asignatura has sacado mejor nota y encima has aprendido más que en las demás... y es que esta observación la hacíamos una vez dos compañeros al final del curso... pues qué más podemos pedir (GE).

Entienden que la eficacia del modelo reside en la exigencia de compromiso colectivo y la cultura cooperativa. Pensaban en su experiencia y en el mismo momento del habla, lo trasladaban a su futuro profesional: "Tengo que adaptarme un poco a lo que tengo en clase, si tengo gente que no tiene cultura cooperativa pues tendré que dedicar un mes a trabajarla para luego poder hacer esto" (GA).

Mayor aceptación de la evaluación colaborativa con el profesor que entre iguales

Coincidimos con Dochy et al. (2002), cuando llegan a la conclusión mediante una revisión bibliográfica de diferentes investigaciones, en que el alumnado se muestra motivado cuando se le presenta nuevas formas de evaluación. En nuestro análisis, destacamos que consideran positivas las técnicas de evaluación y calificación que requieren la valoración personal (autoevaluación o autocalificación):

Mf - Y éramos nosotros mismos quienes nos evaluábamos.

$\mathrm{M} 3$ - Si, si, es que es eso.

M1 - Y si eres un poquito crítico contigo mismo...

Mf - Te enseña a aceptar un poco tus carencias y tus... para intentar mejorar. (GA).

La autoevaluación lleva a una mayor reflexión sobre el propio trabajo y a una mayor responsabilidad por el propio aprendizaje de cada alumno:

Y que además estamos más de acuerdo, porque nosotros sabemos nuestro esfuerzo, porque además es que hay que trabajar todos los días. Entonces tú sabes que te lo estás currando o no, y que si te lo curras más vas a tener más nota y si te lo curras menos pues menos nota. Y entonces al final llega la evaluación y tú sabes más o menos lo que vas a tener y además estás de acuerdo. No como en los exámenes teóricos (GA). 
Así mismo, también consideran muy positivas las técnicas de valoración con el profesor (evaluación compartida, calificación dialogada, etc.): "Eso me encantó, las entrevistas también, porque dan mucha información tanto del alumno como de tu profesor que te está evaluando" (GE). Coinciden ambos en que se conocen mejor alumno y profesor, algo ya verificado (López et al., 2007):

En el momento en que tú te pones a hacer una co-evaluación, me parece que se llamaba, o sea una evaluación o una reunión en la que tú hablas con tu maestro y estás evaluándote a ti con él, y estás teniendo una charla... En ese sentido sabes que, o solamente con ese gesto sabes que está intentando saber cómo ha sido el proceso (GA).

Sin embargo, en sus discursos, la evaluación entre pares no la ven tan positiva, derivado de experiencias personales:

Los compañeros cuando evaluaban no sabían... o sea, evaluar bien a los compañeros, o sea, que evaluaban cosas que no había que evaluar, por ejemplo cuando se hacía un trabajo, evaluar el trabajo y dar explicaciones de cuando se ponía la evaluación por qué no te ha parecido bien, qué es lo que se puede mejorar, y muchas veces era echar tierra a trabajos de los demás por echarlos por tierra. Eso es lo que yo creo que habría que mejorar (GE).

Aportan algunas propuestas de mejora, como la necesaria clarificación de las pautas de evaluación desde el primer momento para saber lo que el profesor espera y evitar problemas de evaluaciones entre pares. Ya lo planteaba Bretones (2008) al preguntarse si la participación de los estudiantes de Educación Superior en su evaluación es objetiva y precisa. Tras una revisión bibliográfica, una de sus conclusiones principales es que aunque existe una leve tendencia a que el alumnado sobrevalore su trabajo, se puede alcanzar una mayor precisión utilizando escalas de evaluación o criterios negociados de valoración:

Entonces, claro, también existe ese problema el desconocimiento de cómo se debe evaluar pues hace que la evaluación la haga a su estilo y sea totalmente particular, y que muchas veces no coincidan ¿no? ... que es lo que hablábamos de la desinformación y que no se sabe lo que te evalúan... muchos no saben lo que están evaluando, o sea, o no saben realmente cuál es el sistema con el que evalúan muchas veces (GA).

La bibliografía considera que la evaluación entre pares puede ser valiosa pues los estudiantes se sienten más involucrados en el proceso y la consideran suficientemente justa y exacta (Dochy et al., 1999). Navarro, Santos, Buscà, Martí- 
nez y Martínez (2010), llegan a la conclusión tras un estudio de casos colectivo (en 60 asignaturas) que pueden surgir conflictos a la hora de proceder a la evaluación entre pares, y que suelen vincularse a la inexperiencia del alumno ante la aplicación de cualquier tipo de evaluación formativa y a la falta de una cultura de evaluación compartida.

Del dicho al hecho hay mucho trecho. Demanda de coherencia entre el discurso pedagógico y la práctica educativa

Todos diferencian constantemente el decir del hacer, vista su experiencia. Y dado que estamos formando futuros formadores, es clave esta disonancia real, especialmente en un modelo que, en contraposición al tradicional, valora el feedback:

Aquí nos ha pasado, te manda hacer trabajos pero luego esos trabajos se quedan ahí, o sea no haces nada con ello ni el niño me entiende por qué lo ha hecho mal o tú no mejoras o no intentas ver qué has fallado ni nada, eso no va a ser formativo, no a va tener carácter formativo, va a ser continua y sumativa (GA).

López et al. (2007) ya apuntaban la necesaria coherencia entre lo que los docentes dicen y lo que hacen, pues consideran que los alumnos aprenden más mediante el ejemplo que mediante los discursos. Nuestros resultados apuntan a la misma idea, ya que se exige que el profesorado "predique con el ejemplo":

Se supone que tendríamos saber trabajar en grupo ¿no? (la mayoría asiente), o sea que es de lo principal que nos enseñan aquí, o debería. Y son ellos los primeros que no lo están haciendo bien, cómo quieren que nosotros aprendamos a trabajar en grupo si desde aquí no lo están haciendo...pues eso, que cada maestro lleva su temario, para algo está la libertad de cátedra... (GA).

La teoría es preciosa, muy bonita (risas), la adornan muy bien... pero luego aquí, en la universidad, y yo creo que eso debería ser en toda la etapa escolar, creo que no se hace y creo que no se tiene en cuenta al individuo (GA.).

Esta demanda enlazaba en los discursos con las dificultades para una implementación real del modelo: "hay que hablar también de la capacidad de los maestros y de los profesores para asumir este sistema" (GA).

La transición de alumno a profesor: Demanda de formación continua para aplicar lo experimentado a la práctica docente

Se ve claramente la importancia de haber experimentado un modelo con el 
que han aprendido más y mejor y su coherente utilización desde el nuevo status de docente: "Todo lo que he trabajado con... lo estoy poniendo en práctica y sí, eso sí que me ha servido mucho” (GE); “...tú tienes ahí una semilla y luego tú ya la alimentas como quieres, pero el caso es tenerla... ¿ ilo hubiera hecho si no lo hubieran hecho conmigo?...y si las has vivido tú, mejor también (asienten varios)... para poder elegir, poder comparar..." (GE).

Se constata incluso cuando se plantean inconvenientes, siendo la carga de trabajo el más citado, algo manifestado ya en multitud de estudios (López et al., 2009):

Hay que hablar también de la capacidad de los maestros y de los profesores para asumir este sistema [...] hay que ver qué profesor está preparado, tiene las condiciones, el tiempo y el interés suficiente como para ser capaz. Porque la evaluación formativa de la que hablamos, o sea la continua, el maestro está corrigiendo constantemente.... Entonces ahí hay que pararse a ver qué ratio de alumnos tiene que tener, si tiene que tener apoyos, si no, si... es que...lo vemos bien pero también está bien si es posible, y yo creo que es posible si el maestro tiene interés y si hay un número de alumnos acorde a la capacidad de trabajo que tiene que tener. Tiene que corregir muchas cosas (GA).

López et al. (2007) afirman que aunque la mayor parte del alumnado prefiere sistemas de trabajo que les supone cierta implicación personal, no dejan de manifestar sus quejas por la mayor carga de trabajo.

Este reconocimiento, desde sus dos identidades, les lleva en ambos casos a consensuar claramente la importancia que tiene la "actitud", verbalizada como "lo vocacional": "Se merece alguien competente, primero porque a mí me gusta mi carrera. Alguien competente es que se preocupa y se forma continuamente, es que es vocacional" (GA); "No te vas a quedar ahí y si te quedas ahí es que te vas a acomodar y claro si estás pensando sólo en cobrar todos los meses" (GA).

Esta transición de alumno a docente alcanza su punto álgido en las constantes referencias, todas ellas críticas, al periodo de prácticas, donde tienen la oportunidad de implementar lo aprendido, y donde más demandan la evaluación formativa:

Entre los egresados, los que tienen experiencias satisfactorias con el modelo lo han intentado implantar en los centros donde desempeñan su actividad profesional, encontrando numerosas barreras: "hay gente muy reacia, sobre todo yo veo que gente más mayor, como que es muy reacia a que se metan en su trabajo o les cambien la forma de trabajar, eso es lo más complicado" (GE).

Plantean todos la necesidad de contar con apoyo y asesoramiento continuo para implantar este nuevo paradigma en la práctica profesional diaria: "que no se perdiese el contacto, quizá que se pudiera de algún modo segur manteniendo, a lo mejor dudas, o experiencias" (GE). 


\section{CONCLUSIONES}

Los discursos analizados nos han mostrado la apuesta por la evaluación formativa, demandando mejoras concretas, especialmente en torno a la evaluación entre pares y a una implementación. Desean explícitamente, dada su satisfacción con el mismo, una ampliación del modelo tanto al prácticum como a su posterior práctica docente.

Entendemos que hay que seguir profundizando en el análisis de los discursos de los propios alumnos, concretando mucho más los debates en torno a aspectos como los señalados. Sería interesante hacerlo especialmente en el discurso de los alumnos y egresados menos favorables a este nuevo paradigma para ahondar en las fisuras del modelo y las mejoras necesarias en el mismo. Resultaría también interesante recorrer dos nuevos caminos que indican los discursos analizados, abriendo nuevas líneas de investigación-acción respecto al prácticum, y contrastando las posibilidades de establecer redes para una formación continua.

\section{AGRADECIMIENTOS}

Este artículo forma parte del resultado del proyecto de investigación titulado: "La docencia universitaria ante el Espacio Europeo de Educación Superior (EEES). Análisis de la situación actual en la Formación Inicial del Profesorado y desarrollo de propuestas basadas en el sistema ECTS y el desarrollo de competencias profesionales", subvencionado por la Junta de Castilla y León, en la convocatoria pública para el desarrollo de proyectos de investigación (2008-2010).

\section{REFERENCIAS}

Álvarez, V., García, E., Gil, F., Romero, S., y Correa, J. (2002). Enseñanza en la Universidad: percepciones de profesores y alumnos. Revista de Educación, 328, 303-323.

Arribas, J. M., Carabias, D., y Monreal, I. (2010). La docencia universitaria en la formación inicial del profesorado. El caso de la escuela de Magisterio de Segovia. Revista Interuniversitaria de Formación del Profesorado, 13(3), 27-35.

Brown, S., y Glasner, A. (2003). Evaluar en la universidad. Problemas y nuevos enfoques. Madrid: Narcea.

Buscá, F., Pintor, P., Martínez, L., y Peire, T. (2010). Sistemas y procedimientos de Evaluación Formativa en docencia universitaria: resultados de 34 casos aplicados durante el curso 2007-2008. Estudios sobre educación, 18, 255276.

Bretones, A. (2008). Participación del alumnado de Educación Superior en su evaluación. Revista de Educación, 347, 181-202.

Capllonch, M., Buscà, F., Martín, M., Martínez, L., y Camerino, O. (2008). Trabajo docente en equipo y evaluación formativa. Redes de trabajo y trabajo en 
red en Educación Física. Revista de la Facultad de Ciencias de la Educación, 8, 219-234.

Conde, F. (2010). Análisis sociológico del sistema de discursos. Cuadernos metodológicos, 43. Madrid: Centro de Investigaciones Sociológicas.

Dochy, F., Segers, M., y Dierick, S. (2002). Nuevas vías de aprendizaje y enseñanza y sus consecuencias: Una nueva era de evaluación. Revista de Docencia Universitaria, 2(2), 13-31.

Dochy, F., Segers, M., y Sluijsmans, D. (1999). The use of self, peer and co-assessment in Higher Education: a review. Studies in Higher Education, 24(3), 331-350.

Gómez, M. C., y Grau, S. (2010). Evaluación de los aprendizajes en el Espacio Europeo de Educación Superior. Alicante: Marfil.

Gros, B., y Romaña, T. (1995). Ser profesor. Palabras sobre la docencia universitaria. Barcelona: Universidad de Barcelona.

Ibarra, M. S., y Rodríguez, G. (2007). El trabajo colaborativo en las aulas universitarias: reflexiones desde la autoevaluación. Revista de Educación, 344, 355-375.

López, V. (2000). Evaluación compartida. Descripción y análisis de experiencias en Primaria, Secundaria y formación del profesorado en el área de Educación Física. Sevilla: M.C.E.P.

López, V. M., Martínez, L. F., y Julián, J. A. (2007). La Red de Evaluación Formativa, Docencia Universitaria y Espacio Europeo de Educación Superior (EEES). Presentación del proyecto, grado de desarrollo y primeros resultados. Revista de Docencia Universitaria, 5(2), 1-19.

López, V. M. (coord.) (2009). Evaluación formativa y Compartida en Educación Superior. Madrid: Narcea.

Navarro, V., Santos, M. L., Buscà, F., Martínez, L., y Martínez, L. F. (2010). La experiencia de la Red Universitaria Española de Evaluación Formativa y Compartida: proceso y abordaje. Revista Iberoamericana de Educación, 52(7), 1-12.

Paillé, P. y Muchielli, A. (2003). L'analyse quialitative en sciences humaines et sociales. París: Armand Colin.

Palomares, A. (2011). El modelo docente universitario y el uso de nuevas metodologías en la enseñanza, aprendizaje y evaluación. Revista de Educación, 355, 591-604.

Pérez, Á., Tabernero, B., López, V. M., Ureña, N., Ruiz, E., Capllonch, M., González, N., y Castejón, F. J. (2008). Revista de Educación, 347, 435-451.

Porto, M., y Rodríguez, R. (2009). Evaluación de estudiantes en la universidad de Santiago de Compostela: percepciones de los propios alumnos. Red $U$. Revista de Docencia Universitaria, 3, 1-22

Ros, S. L. (2001). La evaluación formativa en la Universidad a través de Internet. Barañáin (Navarra): Eunsa.

Sanmartí, N. (2007). 10 ideas clave: Evaluar para aprender. Madrid: Graó.

Santos, M. Á. (2000). La escuela que aprende. Madrid: Morata. 
Tejedor, F. J. (Dir.) (1998). Las estrategias utilizadas por los profesores universitarios para la evaluación del aprendizaje de los alumnos. Madrid: CIDEInforme.

Vallés, C., Ureña, N., y Ruiz, E. (2011). La Evaluación Formativa en Docencia Universitaria. Resultados globales de 41 estudios de caso. Revista de Docencia Universitaria, 9(1), 135-158. 
